\title{
Personified and Multistate Camera Motions for First-Person Navigation in Desktop Virtual Reality
}

\author{
Léo Terziman, Maud Marchal, Franck Multon, Bruno Arnaldi, and Anatole Lécuyer
}

\begin{abstract}
In this paper we introduce novel "Camera Motions" (CMs) to improve the sensations related to locomotion in virtual environments (VE). Traditional Camera Motions are artificial oscillating motions applied to the subjective viewpoint when walking in the $\mathrm{VE}$, and they are meant to evoke and reproduce the visual flow generated during a human walk. Our novel camera motions are: (1) multistate, (2) personified, and (3) they can take into account the topography of the virtual terrain. Being multistate, our CMs can account for different states of locomotion in VE namely: walking, but also running and sprinting. Being personified, our CMs can be adapted to avatar's physiology such as to its size, weight or training status. They can then take into account avatar's fatigue and recuperation for updating visual CMs accordingly. Last, our approach is adapted to the topography of the VE. Running over a strong positive slope would rapidly decrease the advance speed of the avatar, increase its energy loss, and eventually change the locomotion mode, influencing the visual feedback of the camera motions. Our new approach relies on a locomotion simulator partially inspired by human physiology and implemented for a real-time use in Desktop VR. We have conducted a series of experiments to evaluate the perception of our new CMs by naive participants. Results notably show that participants could discriminate and perceive transitions between the different locomotion modes, by relying exclusively on our CMs. They could also perceive some properties of the avatar being used and, overall, very well appreciated the new CMs techniques. Taken together, our results suggest that our new CMs could be introduced in Desktop VR applications involving first-person navigation, in order to enhance sensations of walking, running, and sprinting, with potentially different avatars and over uneven terrains, such as for: training, virtual visits or video games.
\end{abstract}

Index Terms-Navigation, Camera Motions, locomotion simulation.

\section{INTRODUCTION}

Navigation is a fundamental task in 3D interaction with Virtual Environments (VE) [3]. Many Virtual Reality (VR) applications require the possibility to navigate in the VE, such as for virtual visits of architectural projects, training applications, or video games.

In order to improve the immersion of the user during a navigation in a virtual environment, different techniques can be used. Real walking motion can for instance be used by means of locomotion interfaces such as treadmills [15]. This provides vestibular and proprioceptive cues which are naturally present during the real human walk [8]. However, such locomotion interfaces are still costly and cumbersome, and can therefore not always be used.

Visual feedback can also influence the perception of navigation and even of self-motion [9]. Several software techniques relying on visual feedback have thus been proposed, in order to simulate or enhance the locomotion in a VE. The Camera Motions (CMs) introduced by Lécuyer et al. [18] simulate and reproduce the oscillations of the eyes and head motions during the walk. The motion of virtual camera (point of view) in the VE during the navigation generates a visual flow similar than that produced by a real walk. Researchers notably showed that such visual effect not only improve the sensation of walking, but also his perception of the traveled distances in the virtual environment [29].

However, the existing CMs models are rather limited. They were exclusively designed for simulating the walk [18]. They have not been applied to other locomotion modes such as for running or sprinting for example. The current CMs do not take into account information related to the physiology of the virtual human (avatar). Indeed, the user would probably expect different CMs depending for instance on

- Léo Terziman is with INSA / INRIA / DGA, e-mail: leo.terziman@inria.fr.

- Maud Marchal is with INSA / IRISA, e-mail: maud.marchal@irisa.fr.

- Franck Multon is with Université Rennes 2 / M2S, e-mail: franck.multon@univ-rennes2.fr.

- Bruno Arnaldi is with INSA / IRISA, e-mail: bruno.arnaldi@irisa.fr.

- Anatole Lécuyer is with INRIA, e-mail: anatole.lecuyer@inria.fr.

Manuscript received 13 September 2012; accepted 10 January 2013; posted online 16 March 2013; mailed on 1 May 2013.

For information on obtaining reprints of this article, please send

e-mail to:tvcg@computer.org. the size, age or training status of the avatar.

Therefore, in this paper, we study and introduce a new approach which revisits and drastically improves the possibilities offered by Camera Motions in Desktop VR. This approach enables us to display multiple modes of locomotion, and can take into account the properties of the avatar or the topography of the virtual environment. It is based on a locomotion simulator which notably simulates the fatigue and recuperation of the avatar, and handles the synchronization and updates of the camera motions. The two main contributions of our paper are thus:

- A new approach for Camera Motions. We have introduced a generic approach for multistate, personified, and topographydependent Camera Motion in Desktop VR.

- An experimental campaign. We have conducted a series of experiments to evaluate the perception of our new multistate and personified CMs by naive participants when navigating in VE in a first-person mode.

The remainder of this paper is organized as follows: section 2 provides an overview of the existing sensory feedback techniques for enhancing sensation related to locomotion in VE. In section 3 we present our novel approach for multistate and personified camera motions. In section 5 we present the results of our series of experiments. The paper ends with a general discussion and a conclusion.

\section{Related Work}

To augment immersion when navigating in VE, many techniques have been designed to enhance the sensory feedbacks of the user. During the walk, many senses contribute to the sensation of walking: vestibular, proprioceptive [8], but also visual for example [9].

Some techniques are designed to provide vestibular and proprioceptive information to the user by allowing real motions translated into the VE. Techniques using devices such as treadmills [15] or foot platform [14] can be used for such purpose. Vestibular feedback can also be directly induced using the Galvanic Vestibular Simulation (GVS) [21]. Indeed, using two electrodes placed on the mastoids behind the ears, the GVS temporarily alters the vestibular system by sending small electric impulses. 
During real walks, force and vibrotactile information are also produced at each contact of the steps with the ground. Vibrotactile information can be provided in VE through tiles [32] or shoe-based devices [25]. Using vibrators, these devices can simulate different ground types by producing adapted vibrotactile feedback at each step. Moreover, the vibrotactile feedback can also be associated with visual feedbacks to further enhance the step simulation[30].

Audio feedback also provides important information about the locomotion. Footstep sounds depend on the properties of the ground. Different footstep sounds can be simulated in real time for navigations on snow, concrete, wood, gravel and water [22]. Moreover, existing footstep sounds can be modified in real time to change the rendered properties of the ground [28].

Visual feedback is also essential during navigation. Indeed, the visual flow influences the perception of self-motion [9]. Moreover, the Camera Motions [18] improve the immersion during the navigation by simulating the visual flow produced by the oscillations of the point of view during the walk. Camera Motions can also increase the perception of short distances in VE [29]. Compensation of the head orientation to focus the gaze on the objects can also be added to CMs [11] to focus the camera on the objects in the VE. Moreover, CMs can also be used to suggest slopes: the height, speed and orientation of the camera used together increase the perception of virtual slopes [19].

Camera motions are commonly found in many first-person Desktop VR applications or video games [4]. However, the simulated motions are always very simple and are not adapted to the type of locomotion. The only notable exception is Mirror's Edge videogame in which complex precomputed CMs are adapted to a wide range of actions.

\section{Multistate and Personified Camera Motions}

In order to improve the sensation of walking of the users in Desktop VR, we introduce a new approach of Camera Motions.

Our CMs are adapted to the different modes of the human locomotion. In particular, our new camera motions render the walks, runs and sprints. Moreover, we propose new CMs adapted to the physiology of the virtual human. Indeed, the visual feedback of CMs is different if the virtual human is heavier or is in a better physical condition for example. Finally, our CMs are adapted to the relief of the VE.

In this section, we recall the basic principles and components of CMs. We describe next our new CMs. Finally, we detail the improvement made to the CMs to allow multistate, personified and slope dependent simulation.

\subsection{Overview}

The CMs are defined as motions of the virtual camera in the VE used to simulate and enhance the sensation of walking. These motions can be described as a combination of translations $T=\left(T_{x}, T_{y}, T_{z}\right)$ and rotations $R=\left(R_{x}, R_{y}, R_{z}\right)$ around the three different axes of space. The translations can be composed of oscillations $O$, or vibrations $V i b$. Therefore, if the advance speed is defined as $v$ and $H_{\text {eyes }}$ is the height of the eyes of the virtual human, the global equation of CMs is described Equation 1.

$$
\left\{\begin{aligned}
T_{x} & =O_{x} \\
T_{y} & =O_{y}+V i b+H_{\text {eyes }} \\
T_{z} & =O_{z}+v
\end{aligned}\right.
$$

\subsection{Existing Models of Camera Motions}

Previous Camera Motions [18] simulate the movements of the user's head during a locomotion in first person mode by oscillating the position of the camera. The motions of the virtual camera along the three different axes simulate the motions of the viewpoint of the user during the navigation. Previous models of $\mathrm{CMs}$ [18] propose oscillating the camera along the three different axes, producing a complex trajectory. These oscillating motions correspond to the mathematical description of Equation 2, where $T$ is a constant period for the oscillations, $t$ is the current simulation time and $A_{x}, A_{y}$ and $A_{z}$ are constant amplitudes for each axis set from biomechanical data [27].

$$
\left\{\begin{array}{l}
O_{x}=A_{x} \cdot \cos \left(\frac{\pi}{T} t+\frac{\pi}{2}\right) \\
O_{y}=A_{y} \cdot \cos \left(\frac{2 \pi}{T} t\right) \\
O_{z}=A_{z} \cdot \cos \left(\frac{2 \pi}{T} t+\frac{\pi}{2}\right)
\end{array}\right.
$$

A recent technique introduced by Terziman et al. [30] considers other motions of the camera to simulate the impact of the feet with the ground at each step. This technique, also called the "King-Kong Effects" [30], uses high frequency vibrations of the camera (Equation 5) to simulate the contact of the heels and toes of the virtual human with the ground in the VE (Figure 1). The vibrations are produced using a rigid contact model [24].

$$
V i b=A_{\text {event }} \cdot e^{-T_{\text {event }} \cdot \Delta t} \sin (\omega t)
$$

$\Delta t$ is the time between the contact of the heel and toes and $A_{\text {event }}$ and $T_{\text {event }}$ depend of the gait events such as heel and toes contacts [5] (Table 1). $\omega t$ is the frequency of the vibrations, depending of the ground material. We chose this model with $\omega=67 \mathrm{~Hz}$ corresponding to a ground made of wood.

\begin{tabular}{lrr}
\hline Parameters & Heel contact & Toes contact \\
\hline$A_{\text {event }}$ & 0.05 & 0.03 \\
$T_{\text {event }}$ & 2.0 & 1.0 \\
\hline
\end{tabular}

Table 1: Parameters of the vibration model.

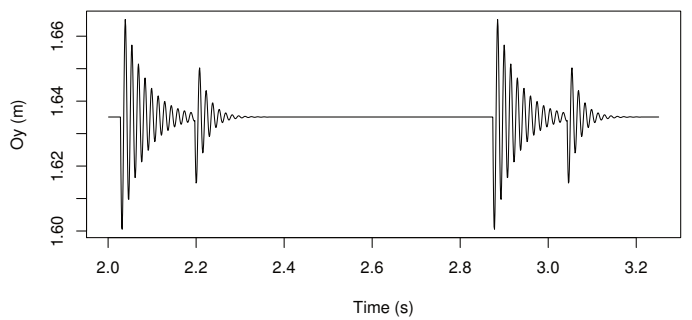

Fig. 1: Vibrations applied to the virtual camera at each step to simulate contacts of the feet with the ground [30].

The two CMs techniques can be combined to further improve immersion and self-motion sensation [30].

\subsection{A Novel Model of Camera Motions}

We propose a new model for multistate and personified CMs. Our model is inspired from the previous work presented in [18] and [30]. However, our new CMs introduce new locomotion modes (running and sprinting) and are adapted to the physiology of the virtual human and the slope of the VE. The new equations of the proposed Camera Motions are detailed in Equations 4 and 5.

$$
\left\{\begin{array}{c}
O_{x}=A_{x} * A_{\text {state }_{x}} \cdot A_{V H_{x}} \cdot \sin \left(\pi P_{\text {step }}+\pi \varphi\right) \\
O_{y}=A_{y} * A_{\text {state }_{y}} \cdot A_{V H_{y}} \cdot \sin \left(2 \pi P_{\text {step }}-\frac{\pi}{2}\right) \\
O_{z}=A_{z} * A_{\text {state }_{z}} \cdot A_{V H_{z}} \cdot \sin \left(2 \pi P_{\text {step }}\right) \\
V i b=A_{\text {vib }} \cdot A_{\text {event }} \cdot e^{-T_{\text {event }} \cdot \Delta t} \sin (\omega t)
\end{array}\right.
$$

To produce multistate CMs, the $A_{\text {state }}$ and $A_{\text {event }}$ parameters are used. Moreover, the personified component of our CMs is defined by the $A_{V H}$ and $A_{v i b}$ functions. $A_{V H}$ and $A_{v i b}$ are the contributions of the 
physiology of the virtual human on the amplitude of the oscillations and vibrations of the camera respectively. Parameter $\varphi$ is the phase between the left or right feet motions. Finally, the previous $T$ and $t$ parameters of the CMs are replaced to synchronize the visual feedbacks with the steps of the virtual human $P_{\text {step }} . P_{\text {step }}$ is defined as the percentage of completion of the current step (Equation 6), $d_{\text {step }}$ is the duration of the current step and $t_{\text {step }}$ the duration since the beginning of the step. Therefore, the new CMs are also synchronized with the advance speed $v$.

$$
P_{\text {step }}=\frac{t_{\text {step }}}{d_{\text {step }}}
$$

\subsection{Personified Camera Motions}

CMs are adapted to the physiology of the virtual human. The feedback depends on both the physical properties of the virtual human, such as its size or weight, and the physical condition of the virtual human. Moreover, the fatigue of the virtual human also influences the visual feedback produced.

\subsubsection{Influence of the Physical Condition of the Virtual Human}

Trained athletes learn to stabilize their head during the run or sprint in order to minimize the energy loss at each step [6]. This effect was simulated by applying coefficients to the amplitude depending on the training status $A_{\text {training }}$ of the virtual human. Moreover, the amplitude of the oscillation also depends on the fatigue of the virtual human $P_{\text {fatigue }}$, defined as a percentage of the maximal energy. Therefore, when the virtual human gets tired, its capacities are decreased and the visual feedback is adapted accordingly. To keep the model as simple as possible, we chose to use the same factors on each axis, i.e. $A_{V H_{x}}=A_{V H_{y}}=A_{V H_{z}}=A_{V H}($ Table 2).

$$
A_{V H}=A_{\text {training }} \cdot\left(\frac{1}{2} P_{\text {fatigue }}+1\right)
$$

\begin{tabular}{lrrrrr}
\hline Fitness & Poor & Fair & Good & Excellent & Superior \\
\hline$A_{\text {training }}$ & 1.0 & 0.8 & 0.6 & 0.4 & 0.2 \\
\hline
\end{tabular}

Table 2: Amplitude factors depending on virtual human training status.

To render fatigue we propose to use a new visual effect. The idea is here to display fatigue as a loss in head stabilization. A roll effect $R_{z}$ is applied on the camera depending on the level of fatigue. The roll is synchronized to locomotion using the phase $\varphi$. The roll of the camera in degrees is given by:

$$
R_{z}=\frac{1}{2} P_{\text {fatigue }} . \sin \left(\pi P_{\text {step }}+\pi \varphi+\frac{\pi}{2}\right)
$$

Thus, the physical condition of the virtual human is rendered by the CMs, depending on its general training status, as well as its level of fatigue $P_{\text {fatigue }}$ (section 3.4.3). Moreover, the CMs also depend on the physical properties of the virtual human.

\subsubsection{Influence of the Physical Properties of the Virtual Human}

Our visual feedback also depends on the physical properties of the virtual human. Currently, our feedbacks render the age, gender, weight and size of the virtual human. All these parameters are used to render a locomotion that depends on the virtual human physiology.

Size. The position of the point of view depends directly on the size of the virtual human. The average height of the eyes was set using anthropometric tables [20]. Moreover, the size is also used in the locomotion simulation to determine the maximal step length [1][31]. Hence the virtual human size can also be perceived through the length of the oscillations of the camera. The height of the camera was set to $H_{\text {eyes }}=0.934 *$ Size. Moreover, the size also influences the relation between step length and step frequency for navigations depending on the advance speed [17][31].
Weight. The weight of the virtual human has an important impact on the perception of the steps. Indeed, the weight can be perceived through the gait of the virtual human. Thus, we propose a new metaphor in which a heavier virtual human would produce more intense vibrations of the camera when its feet are touching the ground. Therefore, the visual vibration of the camera is adapted based on the weight of the virtual human. A coefficient $A_{v i b}$ is applied to the amplitude of the vibration such as:

$$
A_{\text {vib }}=\text { weight } / 100
$$

Age and Gender. Age and gender are displayed through the physical capacity of the virtual human $C_{V H}$ and thus these parameters strongly influence the determination of the advance speed and fatigue. For example, the gait of an athlete is very different from the one of an old lady: the steps are longer and faster.

The physical capacity of the virtual human $C_{V H}$ is inspired from the maximal oxygen consumption $\mathrm{VO}_{2}$ max model [33]. Indeed, our model computes the physical capacity of the virtual human based on the relation between aerobic locomotion and spent energy. The value of $\mathrm{VO}_{2}$ max depends on the gender, age and weight [16]. A value of $C_{V H}$ can be estimated using tables designed to classify participants in different classes of training status [10]. Therefore, our estimation of $C_{V H}$ depends on the gender, age, weight and training status of the simulated virtual human.

\subsubsection{Energy Expenditure Estimation}

The fatigue of the virtual human is also an important factor to render personified CMs. We introduce $P_{\text {fatigue }}$ as the percentage of fatigue, defined in Equation 10, with $E$ the energy available and $E_{\max }$ the maximal amount of energy.

$$
P_{\text {fatigue }}=\frac{E_{\max }-E\left(t_{n}\right)}{E_{\max }}
$$

Therefore we propose the simple following model in which the variation of energy is function of time and effort:

$$
E\left(t_{n}\right)=E\left(t_{n-1}\right)+\alpha\left(P_{C_{V H}}\right) \Delta t
$$

where $E\left(t_{n}\right)$ is the energy available at the time $t_{n}$ and $\Delta t$ is the time interval between $t_{n}$ and $t_{n-1} . P_{C_{V H}}$ is the user input corresponding to the required advance speed. $P_{C_{V H}}$ is defined as a percentage of the physical capacity of the virtual human $C_{V H}$.

The function $\alpha\left(P_{C_{V H}}\right)$ must simulate fast losses of energy at high speeds, but it must also allow recuperation during rest or slow walks. Therefore, we propose two different models for $\alpha\left(P_{C_{V H}}\right)$ depending on the range of $P_{C_{V H}}$. If $P_{C_{V H}}>0.8$, the locomotion requires more than $80 \%$ of the aerobic capacity of the virtual human, and therefore the virtual human would get tired quickly [2]. Accordingly, $P_{C_{V H}}<0.8$ simulates locomotions where the virtual human does not use much energy and therefore restores its energy.

Fatigue of the Virtual Human. For high speeds, i.e. for values of $P_{C_{V H}}>0.8$, the virtual human spends a lot of energy and therefore gets tired rapidly. The time to exhaustion $t_{\text {lim }}$ is used to quantify the amount of energy used for a locomotion at a given speed, depending on the capacity of the virtual human. The experimental results presented by Blondel et al. [2] exhibited a trend which can be modeled by a generalized linear model. The variable $C_{V H}$ was chosen to match the properties of the maximal oxygen consumption model.

First, a Box-Cox transformation showed that the data should be transformed with a logarithm. Therefore, $\log \left(t_{l i m}\right)$ was analyzed depending on $P_{C_{V H}}$ :

$$
\log \left(t_{\text {lim }}\right)=a \cdot P_{C_{V H}}+b
$$

where $a=-5.01$ and $b=10.99$. Thus, if $P_{C_{V H}}>=0.8$ :

$$
\alpha\left(P_{C_{V H}}\right)=-E_{\text {max }} \cdot e^{-a \cdot P_{C_{V H}}-b}, \text { for } P_{C_{V H}}>0.8
$$

After a leave-one-out cross validation on the subject, the adjusted coefficient of determination was $R^{2}=0.85$. 
Recuperation of the Virtual Human. For values of $P_{C_{V H}}<0.8$, the simulator must also simulate the energy recuperation during rest and slow walks. Realistic recuperation times are of the order of magnitude of minutes or even hours depending on the type of exercise performed [7]. Such realistic simulation was found not adapted to our context of virtual navigations. To complete the energy consumption simulator, we propose a new recuperation model designed for VR applications for lower values of $P_{C_{V H}}$. Indeed, our simulator must allow fast energy recuperation at rest and $\alpha\left(P_{C_{V H}}\right)$ must be strictly decreasing. The simulator is the following:

$$
\begin{gathered}
\alpha\left(P_{C_{V H}}\right)=X_{1} \cdot e^{-X_{2} \cdot P_{C_{V H}}+X_{3}, \text { for } P_{C_{V H}}<0.8} \\
\left\{\begin{array}{l}
X_{1}=\frac{E_{\max }\left(\frac{1}{T_{\text {recup }}}+e^{-a . P_{C_{V H}}-b}\right)}{1-e^{-R_{\text {recup }} \cdot P_{C_{V H}}}} \\
X_{2}=R_{\text {recup }} \\
X_{3}=\frac{E_{\text {max }}}{T_{\text {recup }}}-X_{1}
\end{array}\right.
\end{gathered}
$$

where $X_{1}, X_{2}$ and $X_{3}$ are determined by the time to fully regain all the energy at rest $T_{\text {recup }}$. Moreover, the continuity of the function $\alpha\left(P_{C_{V H}}\right)$ must be ensured for $P_{C_{V H}}=0.8$. However, it is mathematically impossible to also ensure the continuity of the first derivative. Thus one constraint is lacking to be able to solve this system. To avoid this problem, the value of the recuperation rate $R_{\text {recup }}$ was chosen manually.

For the evaluation of the simulator, we used $T_{\text {recup }}=10 \mathrm{~s}$ and $R_{\text {recup }}=10$. These values of the parameters provide a good compromise between a realistic behavior and a relatively short recuperation time.

\subsection{Multistate Camera Motions}

We propose new CMs to render different modes of locomotion. In particular, our model renders feedbacks for the walking, running and sprinting modes. The feedback of the locomotion modes is improved using different parameters. Indeed, the locomotion modes are simulated using the amplitude of the oscillations and the type of vibrations produced at each step.

\subsubsection{Oscillations Amplitude}

The amplitude of the oscillations depends on the locomotion mode. We propose arbitrarily to reduce the oscillations when running and sprinting to increase the sensation of speed in the VE. This effect was modeled by applying coefficients to the amplitude depending on the locomotion mode $\left(A_{\text {state }}\right)$. The same factors are used on the three different axes to reduce the complexity of the model. Therefore, $A_{\text {state }_{x}}=A_{\text {state }_{y}}=A_{\text {state }_{z}}=A_{\text {state }}$. The values used for $A_{\text {state }}$ are presented in Table 3 .

\begin{tabular}{rrrrr}
\hline State & Stop & Walking & Running & Sprinting \\
\hline$A_{\text {state }}$ & 0.0 & 1.0 & 0.5 & 0.8 \\
\hline
\end{tabular}

Table 3: Amplitude factors of oscillating CMs depending on the locomotion mode.

\subsubsection{Vibrations}

The type and timing between the different contact events of the feet with the ground provide useful information on the locomotion mode. For example, the difference between run and sprint is defined as the moment when the foot heels stop touching the ground at each step [23]. Therefore, the vibration model only simulates the contact of the toes with the ground for sprinting, while it also simulates the contact of the heels for the walk and run. Therefore, for the heel contact events, $A_{\text {event }}=0$ if the locomotion mode is sprinting. Moreover, the timings between events are also affected. During the walk, the feet are in contact with the ground $60 \%$ of the gait cycle [23]. However, the feet are in contact only $30 \%$ of the cycle for the run [31]. Thus, the gait events are generated accordingly to the locomotion mode.

\subsubsection{Locomotion Modes}

Different locomotion modes can be simulated with our model. In particular, the simulation can control the three following classical modes: (1) walking, (2) running and (3) sprinting [31]. Based on the user inputs, as well as the energy expenditure estimation, the current and appropriate locomotion mode for the navigation can be automatically identified. If the energy available $E$ is too low for the requested locomotion mode, our simulator will automatically stop the locomotion to allow the virtual human to rest. Therefore, in addition to the three walking, running and sprinting modes, a stopped mode was introduced. Each of the different modes has different biomechanical properties that are simulated.

Moreover, only correct transitions between locomotion modes are allowed. Therefore, we defined a state automaton to manage the transitions and maintain the simulation in a coherent state (Figure 2). Our simulator selects the type of each new step to minimize the transition time using the breadth first algorithm to find the shortest path between the current state and the requested state.

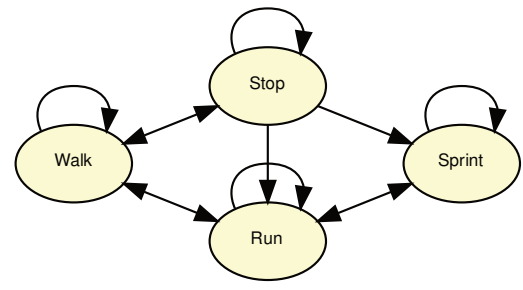

Fig. 2: State Automaton for the transitions between locomotion types.

Finally, the contact events for the heel and toes with the ground are generated depending on the current mode [23]. Therefore, the vibration model is always synchronized with the locomotion.

\subsection{Advance Speed of the Camera Motions}

To further increase the personified and multistate characteristics of our new CMs, we propose to modulate the advance speed $v$ to simulate more accurately the locomotion.

\subsubsection{Advance Speed}

One major difference between the different locomotion modes is the difference in advance speed. The advance speed can be used to render the different locomotion modes. Synchronized CMs match the advance speed produced by the locomotion simulator. Thus, the visual flow is different depending on the locomotion mode. Locomotion modes are defined with non-overlapping speed ranges of user inputs in percentage of the virtual human physical capacity $C_{V H}$. For example, for a 35 years old male of $1.75 \mathrm{~m}$ high and $70 \mathrm{~kg}$ with a good training status, the maximal advance speed for the different locomotion modes on flat ground is given in Table 4.

\begin{tabular}{rrrr}
\hline Stop & Walking & Running & Sprinting \\
\hline $0 \mathrm{~m} \cdot \mathrm{s}^{-1}$ & $1.16 \mathrm{~m} \cdot \mathrm{s}^{-1}$ & $2.79 \mathrm{~m} \cdot \mathrm{s}^{-1}$ & $6.22 \mathrm{~m} \cdot \mathrm{s}^{-1}$ \\
\hline
\end{tabular}

Table 4: Maximal advance speeds set in our implementation for the different locomotion modes for a 35 years old male of $1.75 \mathrm{~m}$ high and $70 \mathrm{~kg}$ with a good training status. The values are set here as consistent with the literature [23].

\subsubsection{Oscillations Phase and Frequency}

The generated steps provide information on the locomotion mode used and the transitions between steps. Indeed, step length and duration depend on the locomotion mode, as well as the virtual human morphology [1][31]. Therefore, synchronization of the CMs with the steps generated by the locomotion simulator improves the rendering of the 
different modes. The oscillation frequency was set to match the current step duration, and incidentally the percentage of completion of the current step $P_{\text {step }}$ (Equation 6). Moreover, the phase of the lateral oscillations $\varphi$ was set depending on the current foot $(\varphi=0.5$ for the left foot and $\varphi=-0.5$ for the right foot).

\subsubsection{Slope-Dependent Camera Motions}

Interestingly enough our new $\mathrm{CMs}$ can also take into account the geometry of the VE. Indeed, the slope has an important impact on the locomotion: the advance speed $v$ directly depends on the slope. When the virtual human navigates down a hill, the advance speed increases, and thus the oscillations of the CMs are also adapted. The inverse is also true when the virtual human climbs a hill.

Moreover, the orientation of the camera also reflects the differences in the slope of the ground. A pitch component $R_{x}$ is introduced in our CMs to improve the perception of the slope. Indeed, the orientation of the camera can also be used to improve the perception of the slope [19]. The camera is oriented to look to the ground in front of the user while climbing or going down a slope. Finally, to avoid jitter on the camera, a low-pass filter is used on the pitch component of the camera with a cut-off frequency of $0.1 \mathrm{~Hz}$.

\subsubsection{Speed Computation}

The navigation in the VE depends actually on the advance speed $v$. The navigation speed is different between walking and running locomotion for example. Moreover, the advance speed depends on the characteristics of the virtual human, i.e. $C_{V H}$ [26].

Our simulator provides a method to evaluate the advance speed in the VE based on the speed required by the user $P_{C_{V H}}$, defined in percentage of the physical capacity of the virtual human $C_{V H}$. For example, a trained athlete will run faster than a man who does not do any exercise. The user would also expect a decrease in velocity when climbing a slope.

The variation of the sum of energies involved during the locomotion $\Delta E_{t o t}$ is null (principle of conservation of the energy):

$$
\Delta E_{t o t}=\Delta E_{c}+\Delta E_{p}+\Delta E_{l o c}+\Delta \varepsilon=0
$$

where $E_{c}$ is the kinetic energy, $E_{p}$ the potential energy, $E_{l o c}$ the energy spent for the locomotion and $\varepsilon$ the sum of the energy lost by the system. Therefore $\Delta E_{l o c}$ is defined such as:

$$
\Delta E_{l o c}=C \cdot m \cdot P_{C_{V H}} . C_{V H} \Delta t
$$

with $m$ the weight of the Virtual Human in $\mathrm{kg}$ and $C=0.336$ a constant required to maintain the compatibility between $C_{V H}$ and the maximal oxygen consumption model.

The speed is directly correlated to the kinetic and potential energy and therefore to the variation of the altitude (i.e. to the slope) and the mass of the virtual human. Moreover, for aerobic locomotions, the advance speed and the oxygen consumption have a direct relation. Our locomotion simulator extrapolates this relation to estimate the advance speed for all the possible locomotions, with $V_{\text {rest }}=3.5$ the energy cost associated with the stopped mode [33]:

$$
P_{C_{V H}} . C_{V H}=3.5 . v+V_{\text {rest }}
$$

However, $\Delta \varepsilon$ is much more complex to evaluate, as it depends on the energy lost in heat or lost during the impacts of the feet with the ground for example. To simplify this problem, the variation of the energy lost was considered to remain nearly constant independently of the slope of the ground.

Finally, the value of the advance speed depends on the slope, the weight of the virtual human, but also all the other physiological parameters used to determine $C_{V H}$. Therefore, the advance speed for the navigation becomes multistate and personified.

\section{Results and Performances}

To evaluate the behavior and performances of our CMs, we generated test cases to exhibit the evolution of the different components of our simulator in various scenarios. For all the test cases, we simulated a 35 years old male of $1.75 \mathrm{~m}$ high and $70 \mathrm{~kg}$ with a good training status.

\subsection{Benchmark}

Our simulator is designed to have an algorithm complexity as small as possible. Indeed, our simulator must be able to run in real time on any kind of platform. Therefore, we need to evaluate the real performances of our simulator in terms of execution time for each frame.

In this scenario, the advance speed was increased linearly from the rest position to the maximal sprint. The navigation was in straight line in a flat VE. The execution time of the CMs feedbacks was measured at each frame. Second, the vibration component of the CMs produced was deactivated. During the two benchmarks, the rendering was set at $50 \mathrm{~Hz}$. The processor used was an Intel Quad Core Extreme Q9300 running at $2.53 \mathrm{GHz}$.

First, we observed that the Mean (M) and Standard Deviation (SD) of the frames execution time was low $(M=159 \mu$ s and $S D=651 \mu \mathrm{s})$. However, the maximal frame execution time was higher $(4941 \mu \mathrm{s})$. The high execution time frames corresponded to the contact of the heel and toes with the ground (Figure 3). We can observe that the time between contacts gets shorter when the speed increases. We also validated that only one event is generated for the sprint.

The first benchmark illustrates that the performances of our simulator are mainly influenced by vibration visual feedbacks. We found that the average execution time without visual vibrations is indeed much lower $(M=5.41 \mu$ s and $S D=1.40 \mu \mathrm{s})$.
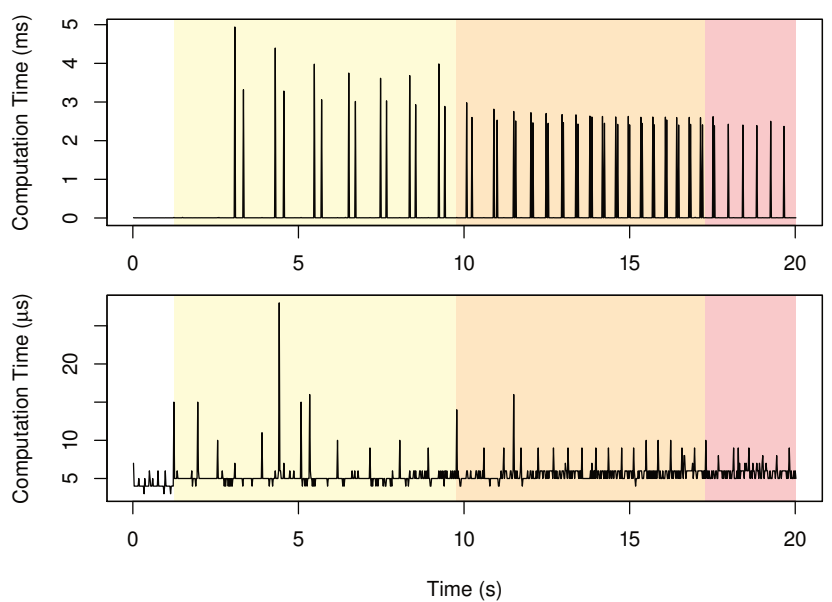

Fig. 3: Benchmark of the locomotion simulator. The computation time of the simulator is measured for each frame with: (a) all feedbacks enabled and (b) without the vibration component. The benchmark is made for a graphical rendering at $50 \mathrm{~Hz}$. The locomotion modes are represented as the background colors, yellow for walking, orange for running and red for sprinting.

\subsection{Influence of Slope}

The slope has a direct impact on the advance speed. Indeed, the advance speed is computed using the variation of potential energy during the step. Therefore, the variations of the speed should follow the inverse of the slope.

The navigation was in straight line, with a constant user input corresponding to a moderate walk (Figure 4). During the navigation, the virtual human crossed a regular $1 \mathrm{~m}$ high and $10 \mathrm{~m}$ wide bump. The advance speed was monitored step-by-step during all the navigation.

During the first flat section, the advance speed remained constant. However, when climbing the bump, the speed decreased proportionally to the slope (Figure 4). On the other hand, when the virtual human started to go down the bump, the speed started to increase proportionally to the slope. Moreover, the speed during this phase was higher than the speed on flat ground. 
Walking speed depending on the slope for a constant input

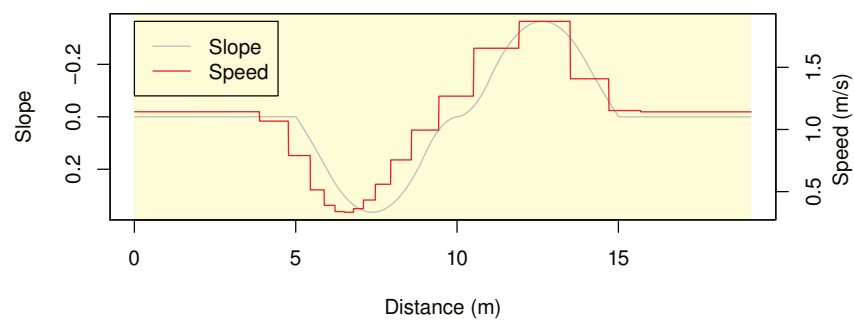

Fig. 4: Influence of the slope on the advance speed. When walking up and down a small hill, the speed depends on the inverse of slope.

\subsection{Influence of Virtual Human Morphology}

The virtual human age, gender, weight and training status have an important impact on many factors of the locomotion simulator. Indeed, these parameters are used to evaluate the fatigue as well as the advance speed. We propose to consider here the influence of the training status on the advance speed. Similar evaluations for the other parameters and/or for the fatigue were performed similarly.

The advance speed increased gradually from the rest position to the maximal sprint (Figure 5). The navigation was in straight lines in a flat VE. Finally, the value of the advance speed was monitored for different values of training status: fair, good and superior.

First, we observed a correct transition between the different locomotion modes from walking to running and finally sprinting. The advance speed strictly increases with the user input (Figure 5). As our simulator produces each step independently, the speed remains strictly constant during a given step. Therefore, the advance speed output is aliased: each level corresponds to a particular step during which the speed remained constant. The speed is lower for the fair training status and greater for the superior training status compared to the good training status. Moreover, the difference between the three values of training status increases with the speed. Indeed, the speed computation is quadratic and not linear. Finally, the step length is not impacted by the different values of training status.

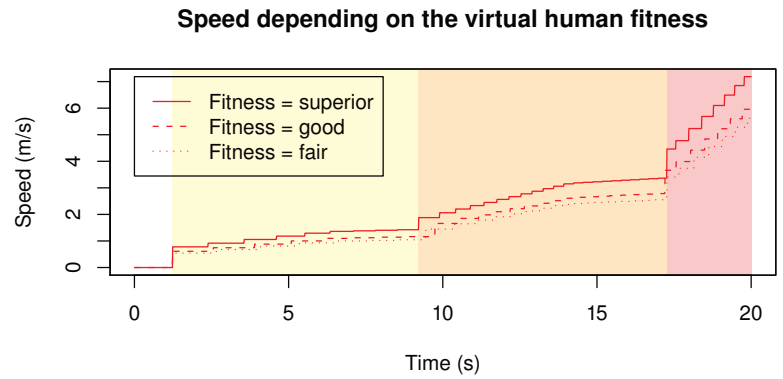

Fig. 5: Influence of the virtual human training status on the advance speed. The locomotion modes are represented as the background colors, yellow for walking, orange for running and red for sprinting.

An example of vertical oscillations produced by our CMs is represented Figure 6 . The produced oscillations seem consistent with data of real walks [12]. The amplitude and frequency of the oscillations ranges are coherent with real data and the vibrations are correctly synchronized with each step.

\subsection{Influence of Virtual Human Fatigue}

The fatigue parameter is essential to the locomotion simulator. Indeed, not only the fatigue is a crucial parameter for our new Camera
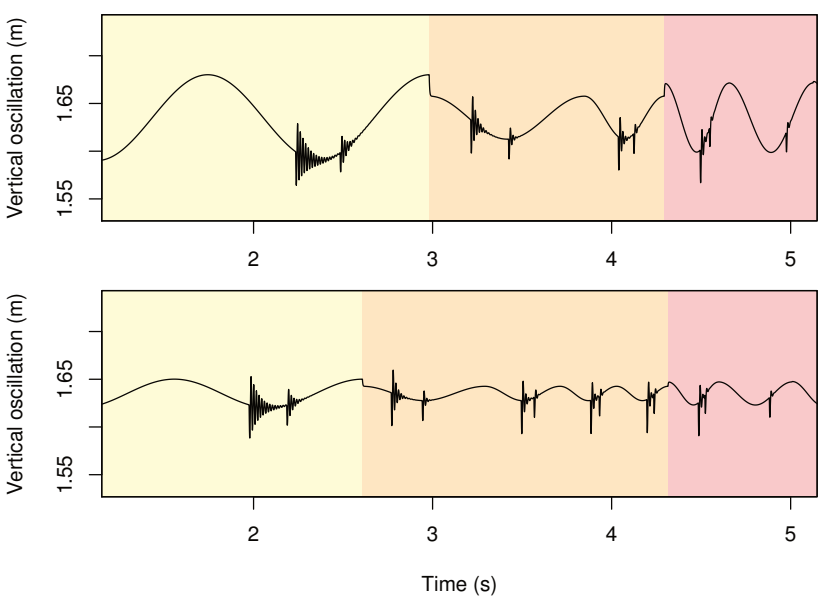

Fig. 6: Vertical oscillations for a 35 years old male of $1.75 \mathrm{~m}, 70 \mathrm{~kg}$ with a good training status (top), and for a 25 years old male of $1.75 \mathrm{~m}$, $70 \mathrm{~kg}$ with a superior training status (bottom). The amplitude and frequency of oscillations, as well as the amplitude of vibrations depend on the locomotion mode. The locomotion modes are represented as the background colors, yellow for walking, orange for running and red for sprinting.

Motions, but it also controls the navigation directly when the energy reserves are depleted.

The user inputs were modulated precisely to exhibit different aspects of the energy expenditure estimation simulator (Figure 7). The simulated navigation was in straight lines on a flat VE. The user inputs consisted in a phase of sprint, followed by a phase of rest. The user then walked at a slow pace and finally ran at a high pace.

During the sprinting phase, the energy decreased very fast, but in a constant manner because the user inputs were constant (Figure 7). When the energy was completely depleted, the virtual human stopped automatically (even when the user continued to ask for sprint), and transition steps were made from sprint to run and then walk. During the resting phase, the energy increased constantly and rapidly. During the slow walk, the energy continued to increase slowly. Finally, during fast running (marathon speed), the energy started to decrease again, but much more slowly than during the sprint. Indeed, the user should be able to run a long time at marathon speed.

Metabolic energy depending on the user inputs on flat ground

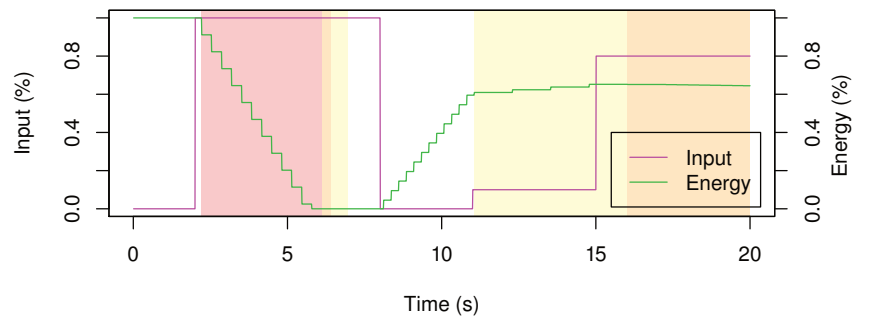

Fig. 7: Influence of the inputs on the virtual human metabolic energy. The locomotion modes are represented as the background colors, yellow for walking, orange for running and red for sprinting. 


\section{Perceptual Evaluation of the Camera Motions}

We conducted a perceptual study of the proposed multistate and personified CMs. Our objective was to demonstrate that CMs allow to perceive the locomotions modes, as well as the characteristics of the virtual human. Our study is composed of four different experiments.

The first experiment is designed to evaluate whether the participants are able to correctly detect the locomotion modes used for the navigation. The second experiment focus on determining whether the transitions between different locomotions modes are perceived correctly. The third experiment seeks to determine if the participants perceive well the properties of the virtual human, such as its training status, weight and age. Finally, the fourth experiment is designed to evaluate the influence of the advance speed on the detection of the locomotion modes.

\subsection{Method}

\subsubsection{Population}

Twelve participants (10 males and 2 females) aged from 20 to 31 (Mean $M=25.75$, Standard Deviation $S D=2.93$ ) performed the three first experiments. Moreover, twelve new participants (11 males and 1 female) aged from 15 to $34(M=25.83, S D=5.1)$ performed the fourth experiment. None of the participants had any known perception disorder. All participants were used to VEs but were naïve with respect to the proposed techniques, as well as to the experimental setup.

\subsubsection{Virtual Environment}

The Virtual Environment was composed of closed room representing a virtual museum with paintings and statues (Figure 8). The room depth was set to $15 \mathrm{~m}$. Moreover, statues were placed along the advance path to improve the visual flow and perception of proportion in the VE. Finally, during the active navigations (third experiment), a bump on the ground was produced using the top of a $10 \mathrm{~m}$ radius sphere. The resulting bump was $1 \mathrm{~m}$ high and $8.7 \mathrm{~m}$ long (Figure 8 ). The participants were always exposed to navigations of $10 \mathrm{~m}$.

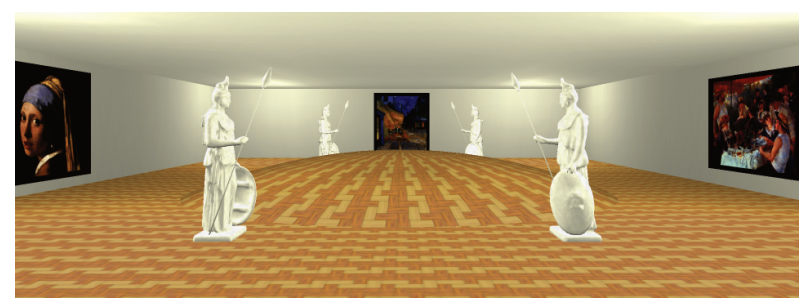

Fig. 8: Virtual Environment.

\subsubsection{Experimental Apparatus}

The participants were seated at $1 \mathrm{~m}$ in front of a 24 inch widescreen monitor with a resolution of $1920 \times 1200$ pixels (physical field of view of $29^{\circ}$ horizontally and $18^{\circ}$ vertically). The rendering was made at a refresh rate of $50 \mathrm{~Hz}$. The participants had the possibility to take breaks by pressing the "Space" key at any time.

\subsubsection{Collected Data}

For the first and fourth experiments, the answers of the participants were recorded at the end of each trial with the answer time. For the second experiment, a boolean was recorded for each trial if the participant detected a transition, as well as the time between the transition and the answer of the participant. Finally, for the third experiment, the preferred trial was recorded.

The participants were also asked to fill a questionnaire at the end of the experiment where they were free to detail their impressions on the different conditions they were exposed to.

\subsection{Experiment 1}

In this experiment, the participants had to detect the locomotion mode used for the navigation (walking, running or sprinting) at various constant advance speeds. We made the hypothesis that the participants would be able to detect correctly the type of locomotion using only the cues provided by our technique. The participant were exposed to passive navigation in straight line. At the end of each navigation, they had to choose which locomotion mode was presented.

\subsubsection{Experimental Conditions}

For each of the possible locomotion modes (Walking, Running, Sprinting), we selected three different speeds corresponding to input ratios of $0.25,0.5$ and 0.75 . It resulted in 9 different possible locomotions. The locomotions were presented randomly by block, each of the condition presented the same number of time than the others. The participants had to choose the correct locomotion mode at the end of the navigation using the keyboard keys " 1 ", " 2 " and " 3 " and then validate their choice with the "Enter" key.

\subsubsection{Results}

We analyzed the Mean (M) and Standard Deviation (SD) of the percentages of correct answer for each locomotion mode. We found that walking was correctly detected $(M=0.95, S D=0.11)$. However, the running and sprinting obtained poor global results $(M=0.26$, $S D=0.20$ and $M=0.34, S D=0.23$ respectively). In order to understand these results, we plotted the mean percentage for each condition (Figure 9). We found that the locomotion modes are underestimated. Indeed, the running locomotions were often detected as walking, and the sprinting as running.

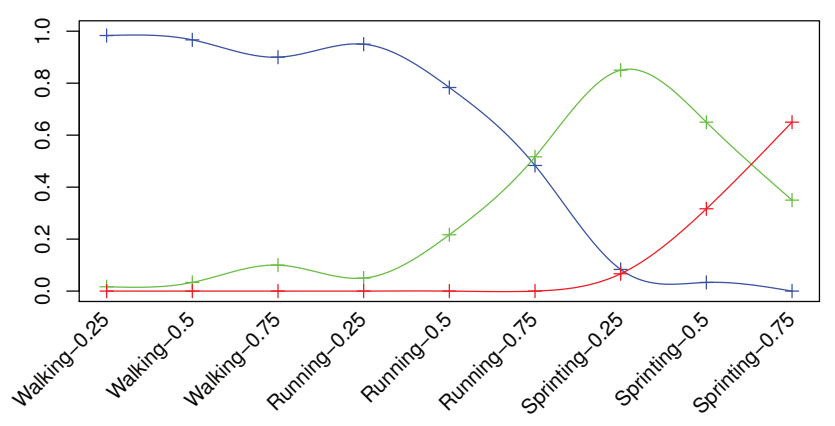

Fig. 9: Detection rates for the three locomotion modes (walking in blue, running in green and sprinting in red).

\subsection{Experiment 2}

During the second experiment, the participants were asked to detect the possible transitions between two locomotion modes for given variations in the advance speed. We made the hypothesis that the participants would be able to detect the transitions between the locomotion modes and not only the variations of the advance speed.

\subsubsection{Experimental Conditions}

For each of the possible locomotion modes (Walking, Running, Sprinting), we selected two different speeds corresponding to inputs of 0.4 and 0.8 percents of the maximal advance speed for each locomotion mode. We tested all the possible combinations between speeds, resulting in 22 different possible locomotions. The locomotions were presented randomly by block, each of the condition presented the same number of time than the others. The participants had to press the "Enter" key at the moment they detected the transition between two locomotion modes. 


\subsubsection{Results}

We analyzed the Mean (M) and Standard Deviation (SD) of the percentages of correct answer for each couple of locomotion mode(Table 5). We found that changes of speed without changing the locomotion mode are correctly detected $(M=92 \%, 81 \%$ and $69 \%)$. However, the percentage of correct detection tends to decrease when the speed increases. The transitions between running and sprinting modes are also correctly detected $(M=71 \%$ and $M=86 \%)$. However, the transitions between the walking and running modes are not detected by the participants: half of the transitions was not detected ( $M=45 \%$ for each type of transition).

\begin{tabular}{lccc}
\hline & Walking & Running & Sprinting \\
\hline Walking & $0.92(0.12)$ & $0.45(0.30)$ & - \\
Running & $0.45(0.27)$ & $0.81(0.26)$ & $0.86(0.15)$ \\
Sprinting & - & $0.71(0.22)$ & $0.69(0.30)$ \\
\hline
\end{tabular}

Table 5: Mean and Standard Deviation of the percentages of correct answers. The first locomotion modes are represented in the rows and the second in the columns. Direct transitions from walking to sprinting are not allowed by the CMs.

\subsection{Experiment 3}

The third experiment was based on a 2 Alternative Forced Choice (2AFC) paradigm. In this experiment, we made the hypothesis that the participants would be able to perceive correctly some properties of the virtual human. The participants were exposed to pairs of active navigations in straight line using different conditions. At the end of each pair, the participants were asked to chose one navigation between two depending on the parameters of the virtual human. In every case, the participants had to choose between the two navigations. The first criterion was designed to evaluate the perception of the virtual human training status. Finally, the second investigated the influence of the age of the virtual human.

\subsubsection{Experimental Conditions}

We tested two different parameters for the virtual human: (1) the training status and (2) the age of the virtual human. All the other parameters were set to simulate a 35 years old male virtual human of $70 \mathrm{~kg}$ and $1.75 \mathrm{~m}$ height with a good training status. We counter balanced the order of presentation of the different conditions. For each condition, we tested three different values. The pairs of values were presented in random order by block, each of the pair presented the same number of time than the others.

The users used a joystick to control their advance speed. At the end of each pair of navigations, they had to select their preferred navigation using two joystick buttons and then validate their choice with the joystick trigger.

\subsubsection{Results}

For a given pair of conditions, each individual performed 6 comparisons. Under the null hypothesis of equal preference between the two conditions, the number of times an individual preferred the first condition follows a binomial distribution with parameters 10 and 1/2. After standardization, such variable can be approximated by a standard normal random variable. Thus, for each pair of conditions, we tested the presence of a preferred condition using a Student's t-test. The p-values were adjusted with a Bonferroni correction.

Influence of the Virtual Human Fitness. The different values of virtual human training status were: (1) fair $F_{\text {fair }},(2)$ good $F_{\text {good }}$ and (3) superior $F_{\text {sup }}$. The participants had to select the navigation where the virtual human had the best training status. We analyzed the answers of participants for the different training status in order to determine if the training status of the virtual human was correctly perceived by the participants.
The analysis showed that $F_{\text {Sup }}$ was more often significantly chosen than $F_{\text {good }}(t(11)=21.06, p<0.001)$ and $F_{\text {fair }}(t(11)=9.94, p<$ $0.001)$. Moreover, $F_{\text {good }}$ was more often significantly chosen than $F_{\text {fair }}(t(11)=4.21, p=0.002)$. The results are represented in Table 6. Ours results suggest that our personified CMs provide enough feedback to always detect the training status of the virtual human correctly.

\begin{tabular}{lccc}
\hline & $F_{\text {fair }}$ & $F_{\text {good }}$ & $F_{\text {sup }}$ \\
\hline$F_{\text {fair }}$ & - & $0.72(0.28)$ & $0.97(0.10)$ \\
$F_{\text {good }}$ & $0.83(0.22)$ & - & $1.0(0.0)$ \\
$F_{\text {sup }}$ & $0.89(0.30)$ & $0.92(0.15)$ & - \\
\hline
\end{tabular}

Table 6: Mean and Standard Deviation of the percentage of correct answers. The table columns and rows represent the three different training status: fair $\left(F_{\text {fair }}\right)$, good $\left(F_{\text {good }}\right)$ and superior $\left(F_{\text {sup }}\right)$. The first presented conditions are represented in the rows and the conditions presented in second are in columns.

Influence of the Virtual Human Age. The different values of virtual human age were: (1) 25 years old $A_{25}$, (2) 35 years old $A_{35}$ and (3) 55 years old $A_{55}$. The participants had to select the navigation where the virtual human was older.

The analysis showed that $A_{55}$ was more often significantly chosen than $A_{35}(t(11)=8.40, p<0.001)$ and $A_{25}(t(11)=8.40, p<0.001)$. Moreover, $A_{35}$ was almost significantly more often chosen than $A_{25}$ $(t(11)=3.63, p=0.006)$. The results are represented in Table 7. Ours results suggest that our personified CMs provide enough feedback to detect the age of the virtual human correctly.

\begin{tabular}{cccc}
\hline & $A_{25}$ & $A_{35}$ & $A_{55}$ \\
\hline$A_{25}$ & - & $0.58(0.29)$ & $0.83(0.27)$ \\
$A_{35}$ & $0.75(0.21)$ & - & $0.92(0.15)$ \\
$A_{55}$ & $0.97(0.10)$ & $0.89(0.22)$ & - \\
\hline
\end{tabular}

Table 7: Mean and Standard Deviation of the percentage of correct answers. The table columns and rows represent the three different ages: $25\left(A_{25}\right), 35\left(A_{35}\right)$ and $55\left(A_{55}\right)$ years old. The first presented conditions are represented in the rows and the conditions presented in second are in columns.

\subsection{Experiment 4}

The fourth experiment was similar to the first one. However, for the tested navigation, the advance speed was multiplied by various factors in order to determine the impact of the VE on the perception of first person locomotions. Indeed, we made the hypothesis that the detection of the locomotion modes could be improved by improved by adding a factor on the advance speed.

\subsubsection{Experimental Conditions}

Contrary to the first experiment, we added a constant factor to the advance speed to increase the locomotion speed in the VE and improve the perception of the different locomotion modes. Moreover, we also modified the algorithm used to compute the step length for the sprint in order to keep a constant step length independent of the speed factor. Thus the frequency of the steps during the sprint was decreased to be inversely proportional to the speed factor $S F$.

We tested three different speed factors $S F$ of 1,2 and 3. For each speed factor we counter-balanced the order of presentation. Moreover, for each of the possible locomotion modes (Walking, Running, Sprinting), we selected three different speeds corresponding to input ratios of $0.25,0.5$ and 0.75 . It resulted in 9 different possible locomotions. The locomotions were presented randomly by block, each of the condition presented the same number of time than the others. 


\subsubsection{Results}

We analyzed the Mean (M) and Standard Deviation (SD) of the percentages of correct answer for each locomotion mode. We found that walking was correctly detected independently of the speed factor (Table 8). For $S F=1$, the results for the running and sprinting modes are consistent with the first experiment. However, the percentage of correct answer gradually increase with the speed factor. Indeed, the running and sprinting detection are superior with $S F=2(M=36 \%$ $S D=0.31$ and $M=49 \%, S D=0.3$ respectively). Finally, the best results were obtained for $S F=3$ with $M=50 \%, S D=0.27$ for the run and $M=79 \%, S D=0.23$ for the sprint. For each condition we plotted the mean percentage of correct answer (Figure 10). We found again that the locomotion modes are clearly underestimated. Indeed, the running locomotions are detected as walking, and the sprinting as running. However, the underestimation of the locomotion mode clearly decreases when the speed factor $S F$ is increased.

\begin{tabular}{lccc}
\hline Speed Factor & 1 & 2 & 3 \\
\hline Walking & $0.99(0.02)$ & $1.0(0.0)$ & $0.93(0.12)$ \\
Running & $0.22(0.22)$ & $0.36(0.31)$ & $0.50(0.27)$ \\
Sprinting & $0.16(0.17)$ & $0.49(0.30)$ & $0.79(0.23)$ \\
\hline
\end{tabular}

Table 8: Mean and Standard Deviation of the percentages of correct answers depending on the speed factor.
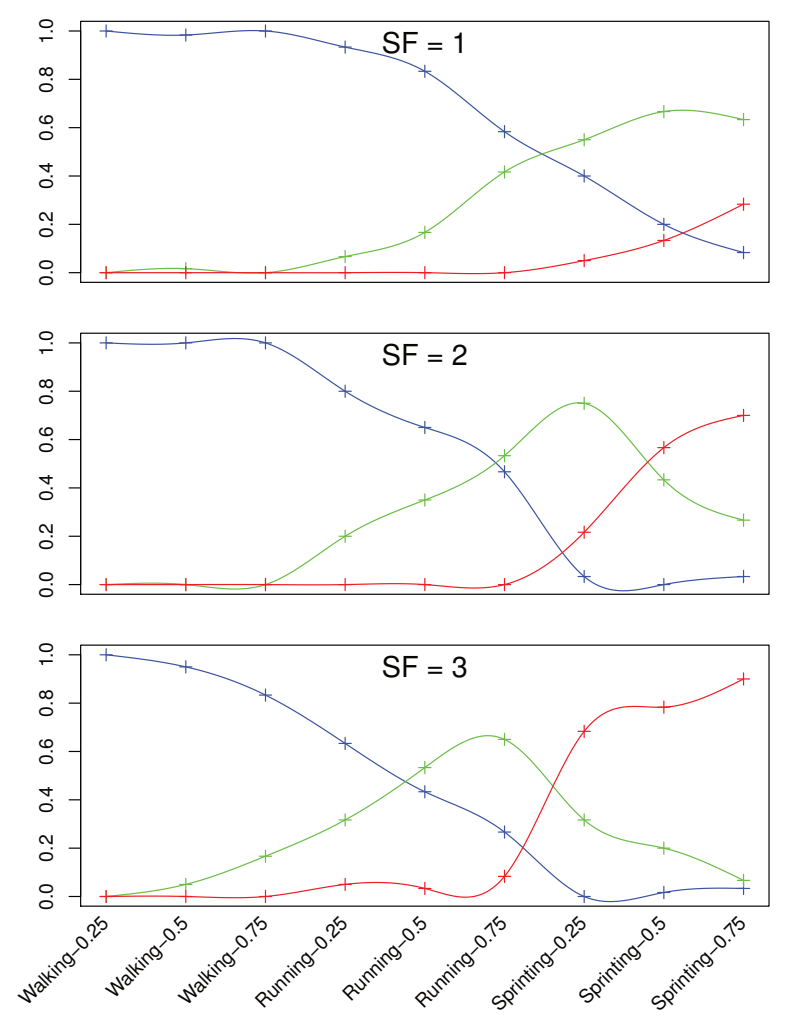

Fig. 10: Detection rates for the first, second and third conditions for the three locomotion modes (walking in blue, running in green and sprinting in red).

\subsection{Conclusion}

To sum up, our novel CMs produce a multistate and personified navigation. The users are able to perceive the properties of the virtual human in first-person mode. Moreover, the different locomotion modes are also detected correctly when using a factor on the advance speed to compensate the underestimation inherent to VR.

\section{Discussion}

Our results suggest that our novel camera motion model, associated with our locomotion simulator, can provide meaningful visual feedback which can be successfully exploited by users to identify the locomotion mode, the transitions between the locomotion modes, and at least two parameters of the virtual human (age and training status).

Interestingly, we have found that the participants globally underestimated the advance speed in the VE. Indeed, running motions were often classified as walking ones, or sprinting motions were classified as running ones. For a "normal" speed factor (of 1), some participants explicitly notified or stressed this effect: "I think the speed should be increased because for me it felt more like slow walk, normal walk and run". Scaling up the advance speed parameter seems to improve the detection rates and reduce the shift. We found that the best detection rates, closest to what would be expected, were achieved for a speed factor of 3. This underestimation might be related to the wellknown underestimation of distances in virtual environments, which would here straightforwardly influence the speed estimation [13].

We also found that participants were able to correctly discriminate the motions corresponding to different values of age or training status of the virtual humans used. To do so, participants could rely on the advance speed but also on numerous other visual cues embedded in the camera motions : changes of locomotion mode, number of contacts with the ground for each feet, amplitudes of the oscillations, etc. For example, the simulated fatigue seems to be an important factor for some participants, as quoted in the subjective questionnaires: "I watched if I could get over the hill in one smooth motion or if I had to slow down or stop". Future work could now focus on evaluating the influence of each visual cue on the resulting perception and estimation.

As observed in the post-hoc questionnaire and as informal feedback, it seems that the participants have well appreciated our new $\mathrm{CMs}$, and even suggested some interesting applications such as video games or virtual visits. Some participants even suggested to create a simulator to preview how walking would "feel" when getting older.

\section{CONCLUSION}

In this paper, we proposed novel Camera Motions for multistate and personified navigation. We proposed a generic approach to display the multiple modes of locomotion, and to take into account the properties of the avatar or the topography of the virtual environment. Our CMs are adapted to walking, running and sprinting locomotions. Moreover, the physiology of the virtual human is taken into account: the locomotion is constrained by the physical capacity of the virtual human which depends on its age, gender, weight and training status. Moreover, the locomotion also depends of the fatigue and recuperation of the virtual human. Finally, our new CMs integrate the topography of the VE to render feedbacks adapted to the slope.

We conducted an experimental campaign composed of a series of experiments to evaluate the perception of our new multistate and personified CMs by naive participants when walking in VE in a firstperson mode. We found notably that participants could discriminate (and perceive transitions) between the different locomotion modes, by relying exclusively on our CMs. They could also perceive some properties of the avatar, like the virtual human training status and age.

Future work could focus first on the technological aspects. Our locomotion simulator could be further improved by considering other physiological or biomechanical properties. Other properties of the VE could also be considered such as the type of ground (e.g. water, snow, sand, etc). Other feedbacks (e.g. auditory or vibratory) could be designed based on our approach and synchronized with our CMs. Second, future work could also focus on new evaluations of the proposed techniques in both desktop and immersive virtual reality. We could notably study the influence of each individual parameter of our locomotion simulator on the perceived motions. The produced motions could also be compared with real head motion data acquired using a tracking system. Our techniques could also be evaluated in more complex virtual environments for instance in outdoor scenes. 


\section{RefERENCES}

[1] R. M. Alexander. Principles of Animal Locomotion. Princeton University Press, 2003. 3.4.2, 3.6.2

[2] N. Blondel, S. Berthoin, V. Billat, and G. Lensel. Relationship between run times to exhaustion at $90,100,120$, and $140 \%$ of $\mathrm{vVO} 2 \max$ and velocity expressed relatively to critical velocity and maximal velocity. International journal of sports medicine, 22(1):27-33, 2001. 3.4.3, 3.4.3

[3] D. A. Bowman, E. Kruijff, J. J. LaViola, Jr., and I. Poupyrev. 3D User Interfaces: Theory and Practice. Addison-Wesley, 2005. (document)

[4] P. G. de Barros, R. W. Lindeman, and T. J. Loughlin. Head-movement evaluation for first-person games. In Proceedings of the 27th international conference on Human Factors in Computing Systems, pages 43994404, 2009. 2

[5] S. A. Dugan and K. P. Bhat. Biomechanics and analysis of running gait. Physical Medicine and Rehabilitation Clinics of North America, 16(3):603-621, 2005. 3.2

[6] M. Fredericson and T. Moore. Core stabilization training for middle-and long-distance runners. New Studies in Athletics, 20(1):25-37, 2005. 3.4.1

[7] C. J. Gore and R. T. Withers. The effect of exercise intensity and duration on the oxygen deficit and excess post-exercise oxygen consumption. European Journal of Applied Physiology and Occupational Physiology, 60:169-174, 1990. 3.4.3

[8] L. R. Harris, M. R. Jenkin, and D. C. Zikovitz. Vestibular cues and virtual environments: Choosing the magnitude of the vestibular cue. In Proceedings of the IEEE conference on Virtual Reality, pages 229-236, 1999. (document), 2

[9] L. R. Harris, M. R. Jenkin, D. C. Zikovitz, F. Redlick, P. M. Jaekl, U. Jasiobedzka, H. L. Jenkin, and R. S. Allison. Simulating self-motion I: Cues for the perception of motion. Virtual Reality, 6(2):75-85, 2002. (document), 2

[10] V. H. Heyward. Advanced Fitness Assessment and Exercise Prescription. Human Kinetics, 5 edition, 2006. 3.4.2

[11] S. Hillaire, A. Lécuyer, R. Cozot, and G. Casiez. Using an eye-tracking system to improve camera motions and depth-of-field blur effects in virtual environments. In Proceedings of the IEEE conference on Virtual Reality, pages 47-50, 2008. 2

[12] E. Hirasaki, S. Moore, T. Raphan, and B. Cohen. Effects of walking velocity on vertical head and body movements during locomotion. Experimental Brain Research, 127(2):117-130, 1999. 4.3

[13] V. Interrante, B. Ries, and L. Anderson. Distance perception in immersive virtual environments, revisited. In Proceedings of the IEEE conference on Virtual Reality, pages 3-10, 2006. 6

[14] H. Iwata, H. Yano, and F. Nakaizumi. Gait master: A versatile locomotion interface for uneven virtual terrain. In Proceedings of the IEEE conference on Virtual Reality, pages 131-137, 2001. 2

[15] H. Iwata and Y. Yoshida. Path reproduction tests using a torus treadmill. Presence: Teleoperators and Virtual Environments, 8(6):587-597, 1999. (document), 2

[16] G. M. Kline, J. P. Porcari, R. Hintermeister, P. S. Freedson, A. Ward, R. F. McCarron, J. Ross, J. M. Rippe, et al. Estimation of vo2max from a onemile track walk, gender, age, and body weight. Medicine and Science in Sports and Exercise, 19(3):253, 1987. 3.4.2

[17] A. D. Kuo. A simple model of bipedal walking predicts the preferred speed-step length relationship. Journal of biomechanical engineering, 123(3):264, 2001. 3.4.2

[18] A. Lécuyer, J.-M. Burkhardt, J.-M. Henaff, and S. Donikian. Camera motions improve the sensation of walking in virtual environments. In Proceedings of the IEEE conference on Virtual Reality, pages 11-18, 2006. (document), 2, 3.2, 3.3

[19] M. Marchal, A. Lécuyer, G. Cirio, L. Bonnet, and M. Emily. Walking up and down in immersive virtual worlds: Novel interactive techniques based on visual feedback. In Proceedings of the IEEE Symposium on $3 D$ User Interfaces, pages 19-26, 2010. 2, 3.6.3

[20] R. Motmans. Dinbelg antropometric tables, 2005. 3.4.2

[21] N. Nagaya, M. Sugimoto, H. Nii, M. Kitazaki, and M. Inami. Visual perception modulated by galvanic vestibular stimulation. In Proceedings of the ACM International Conference on Augmented Tele-Existence, pages 78-84, 2005. 2

[22] R. Nordahl, S. Serafin, and L. Turchet. Sound synthesis and evaluation of interactive footsteps for virtual reality applications. In Proceedings of the IEEE Conference on Virtual Reality, pages 147-153, 2010. 2

[23] T. F. Novacheck. The biomechanics of running. Gait \& Posture, 7(1):77-
95, 1998. 3.5.2, 3.5.3, 4

[24] A. M. Okamura, J. T. Dennerlein, and R. D. Howe. Vibration feedback models for virtual environments. In Proceedings of the IEEE Conference on Robotics and Automation, volume 1, pages 674-679, 1998. 3.2

[25] S. Papetti, F. Fontana, M. Civolani, A. Berrezag, and V. Hayward. Audiotactile display of ground properties using interactive shoes. Haptic and Audio Interaction Design, pages 117-128, 2010. 2

[26] M. E. Pearce, D. A. Cunningham, A. P. Donner, P. A. Rechnitzer, G. M. Fullerton, and J. H. Howard. Energy cost of treadmill and floor walking at self-selected paces. European Journal of Applied Physiology and Occupational Physiology, 52(1):115-119, 1983. 3.6.4

[27] T. Pozzo, A. Berthoz, and L. Lefort. Head stabilization during various locomotor tasks in humans. Experimental Brain Research, 82(1):97-106, 1990. 3.2

[28] S. Serafin, L. Turchet, and R. Nordahl. Extraction of ground reaction forces for real-time synthesis of walking sounds. In Proceedings of the 2009 Audio Mostly Conference, number 4, pages 99-105, 2009. 2

[29] L. Terziman, A. Lécuyer, S. Hillaire, and J. M. Wiener. Can camera motions improve the perception of traveled distance in virtual environments? In Proceedings of the IEEE Conference on Virtual Reality, pages 131-134, 2009. (document), 2

[30] L. Terziman, M. Marchal, F. Multon, B. Arnaldi, and A. Lécuyer. The king-kong effects: Improving sensation of walking in VR with visual and tactile vibrations at each step. In Proceedings of the IEEE Symposium on $3 D$ User Interfaces, 2012. 2, 3.2, 1, 3.2, 3.3

[31] C. L. Vaughan. Biomechanics of running gait. Critical Reviews in Biomedical Engineering, 12(1):1, 1984. 3.4.2, 3.5.2, 3.5.3, 3.6.2

[32] Y. Visell, J. R. Cooperstock, B. L. Giordano, K. Franinovic, A. Law, S. McAdams, K. Jathal, and F. Fontana. A vibrotactile device for display of virtual ground materials in walking. Lecture Notes in Computer Science, 5024:420, 2008. 2

[33] J. H. Wilmore and D. L. Costill. Physiology of sport and exercise. Human Kinetics, 1994. 3.4.2, 3.6.4 